\title{
Models and Algorithms of Formation of Route Transport Network of a City
}

\author{
Sergey Belokurov ${ }^{1}$, Vladimir Belokurov ${ }^{2 *}$, Vladimir Zolnikov², Valery Lavlinsky², and \\ Alexei Shtepa ${ }^{2}$ \\ ${ }^{1}$ Voronezh Institute of the Federal Penitentiary Service of Russia, 1a Irkutskaya Str, Voronezh, \\ 394072, Russia \\ ${ }^{2}$ Voronezh State University of Forestry and Technologies Named after G.F. Morozov, 8 Timiryazev \\ Str., Voronezh, 394087, Russia
}

\begin{abstract}
Mathematical modeling in optimization problems of management of urban passenger transport in large cities is considered. The simulation of route of transportation network of large cities is determined by a relatively large number of their specific and individual properties which, in the process of their functioning, become more complex and interdependent ones. Algorithm of rational route network taking into account annular and circular routes in the schemes of organization of urban passenger transport is proposed.
\end{abstract}

\section{Introduction}

Currently, infrastructure development of major cities and the prospects of improvement allow us to identify a stable trend of increasing role of transport systems that are particularly important in the organization of management of passenger transport. City transport system needs to be adequate to dynamics of its passenger traffic, both at the stages of creation and operation, and in the process of improving the existing route and transport network.

\section{Material and methods}

Models and methods of system analysis and theory of mathematical modeling are the most effective ones to solve this problem, allowing to obtain the best solutions from the many available ones for the specific situation [1,2]. Mathematical methods in finding optimal choices in multi-stage task selection for multi-objective optimization in this case should include enumeration of entire set of possible solutions.

\footnotetext{
*Corresponding author: alexei_shtepa@mail.ru
} 


\section{Theory}

Following criteria can be considered as the main ones in organization of passenger transport:

$$
t_{c} \rightarrow \min , l_{m} \rightarrow \min , n_{m} \rightarrow \min ,
$$

where $t_{c}$ - minimum travel time of passengers; $l_{m}$ - travel distance of passengers; $n_{m}-$ minimum number of passenger vehicles.

For the selected criteria of optimization, possible passenger flow may be considered as a system constraints. Its lower limit will be the totality of served passenger traffic $Q_{j i}=Q_{\min }$, upper limit is allowable number of rolling stock $n_{m} \rightarrow \min$ on the route $m$ and its capacity $q \rightarrow \max$. In this case, restrictions on the size of the passenger flow will have the form $Q_{m}=n_{m} \cdot q=Q_{\max }$.

$$
Q_{\min } \leq Q \leq Q_{\max },
$$

The formation of the route network considering (1) and (2) is made in 2 stages. The first stage involves construction of circular routes, and the second one - pendulum routes. For this we form the graph $G(V, D)$, in which the vertices $V$ are many stopping points (SP) $Q$, and $D$ are arcs connecting the vertices $V$. In this case, you would create the following matrix: average daily passenger traffic $\left\|X_{i j}^{c c}\right\|$ from point $i$ to point $j$; bandwidth of stopping points; the length of routes; and average time of vehicular movement on the route $\left\|t_{i j}\right\|$.

After the formation of source data in schema of organization of urban passenger transport, calculation of rational route network taking into account pendulum and circular routes is made.

The formation of circular routes is carried out using procedures of graph theory $G_{m}\left(V_{m}\right.$, $D_{m}$ ) for every route $m$ and type of transport in several stages [3, 4]:

In the first stage, the search for Hamiltonian cycles in $G_{m}$ is made on the basis of average daily matrix of passenger traffic $\left\|X_{i j}^{c c}\right\|$ using the brute force methods of Roberts and Flores and multichain method of Selby $[11,4]$.

In the second stage when using the method of pyramidal sorting (the algorithm of Williams and Floyd) ranking of routes by increasing average daily traffic is performed.

In the third stage, you select the route $m_{\max }$ with maximum traffic is chosen, on the basis of which all the circular routes are selected, connecting at least by one edge with $m_{\max }$.

The fourth stage assumes inclusion of end points in the final cycles is made on the basis of matrices $\left\|V_{s p}\right\|$ and. Matrix $\left\|V_{s p}\right\|$ defines the number of selected vertices of the cycles, which are the end points.

At the fifth stage for all routes, including $m_{\max }$, for the period of maximum traffic flow calculation for finding the shortest routes between all vertices of the cycle $m_{\max }$ is made with the application of graph $G_{m}$, final points and matrix $[\|n\|],\left\|X_{i j}^{c c}\right\|,\left\|l_{i j}\right\|$. As far as graph $G_{m}$ is considered for each type of passenger vehicles, while generating the constraints, there is the opportunity to go from $[n]$ to the average hourly value $[Q]$, that is

$$
[Q]=[n] \cdot q_{n},
$$

where, $[n]$ - restrictions on the transport capacity of a stopping point on this route; $q_{n}-$ the capacity of considered type of passenger vehicle. 
The number of incoming passenger vehicles at stopping points (SP) depends on the permissible interval of arrivals of passenger vehicles at the SP, which is adopted in the period of maximum traffic flow $J_{\min }=1 \mathrm{~min}, J_{\max }=1 \mathrm{~min}$, that is restriction can be taken:

$$
j_{\min } \leq J_{i} \leq J_{\max }
$$

Since:

$$
J_{i}=\frac{\Delta T_{p . f}}{n} \rightarrow[n]=\frac{\Delta T_{p . f}}{J_{i}},
$$

where, $J_{i}$ - the interval of passenger vehicles arriving at the $i$-th SP; $\Delta T_{p . f}$ - time period of stable passenger flow.

In this case, taking into account (3) and (5,) the limit on the amount of passenger flow through the $i$-th SP would be

$$
[Q]=\frac{60 \Delta T_{p . f . q_{n}}}{J_{i}}
$$

Based on the conditions of appropriateness for minor routes, the condition (2) is stored in the lower limit, that is $Q_{\min } \leq Q_{i}$, and the upper limit may be set by expert.

On the sixth stage the choice of the most efficient route is made $m_{e}$ taking into account bidirectional nature of circular route to build the entire set of routes. In this case we use the main indicators influencing the efficiency of urban passenger transport formed both in the flow of passengers and in their location on the route network of the city. The calculations used data for the time period of maximum traffic flow for each direction, namely: the length of the route $l_{m}$ duration of trip $t_{D}$; the total volume of passenger traffics $\sum Q_{i j}$ maximum passenger numbers for each trip $Q_{\mathrm{m}}^{\max }$; maximum passenger traffic on the route $Q \sum_{m}^{\max }$; required amount of transport on line $n_{m}$. In addition average distance of the passenger trip $l_{\text {p.t. }}$, filling ratio of transport $\gamma$, the period of maximum traffic flow and the coefficient of irregularity of passenger flow along the length of the route during this period is determined.

The choice of best route is made on the basis of certain indicators. And, because it uses a lot of different factors, which complicates the task of routes formation, then indicators are used that mostly affect social and economic aspects of passenger transport.

The task can be simplified if the basic criterion of optimality among the set of proposed routes would be used given hourly production on each route, that is

$$
W_{m}=\frac{\sum Q_{i j} \cdot l_{i j}}{\Delta T_{d a y} \cdot l_{m}}, W_{m} \rightarrow \max ,
$$

where, $Q_{i j}$ - traffic of passengers on the shortest path from point $i$ to point $j ; l_{i j}-$ distance of passenger trips during the traffic $Q_{i j} ; \sum Q_{i j} \cdot l_{i j}$ - passenger flow of the route $m$; $\Delta T_{d a y}$ - daily duration of the route; $l_{m}$ - length of the route.

Introduction of given performance $W_{m}$ is due to the fact that the length of all the routes are different, therefore, the so-called efficient route is introduced $m_{i}\left(W_{m}^{\max }\right)=m_{e}$.

At the seventh stage selected route $m_{e}$ is included in the scheme of RT of the city and it is proposed to consider the expert's recommendation for route acceptation. If the route is accepted by the expert, graph $G_{m}$ is excluded with the entire volume of trips served by 
selected route $m_{e}$, also bandwidth matrix of SP $\|[n]\|$, daily trips $\left\|X_{i j}^{c c}\right\|$ and correspondence with a period of stable passenger traffic $\left\|\left[X_{i j}^{\Delta t}\right]\right\|$ should be corrected. In the case of negative expert decision, $m_{e}$ route is excluded from consideration, going back to all the routes connected with $m_{\max }$, the choice is made from the following most effective $m_{e}$, which is provided to the expert for consideration.

In the eighth stage calculations after consideration of all design options for circular routes are ended. Thus, circular routes with a significant number of passengers do not fall within the scheme of the road network of the city. Circular routes, which, according to the calculations, form a single route network of the city, are excluded from the graph $G$. After that, to complete the formation of unified route network you must go to the technique of forming a plurality of circular routes. The design of circular routes in graph theory is reduced to the task of finding the maximum flow under the given constraints and they are considered as linear programming problems. In this case, the following methods can be used: simplex method (linear programming) [4,5]; Gomori's method [6,12]; STEM method using "man-machine" procedure [9,13]. STEM method, unlike Pareto's method and other techniques, allows, in multivariate tasks, to get satisfactory optimum of minimal solution to constraint system, not using help of decision makers (DM) in the initial stages of calculations [7-10]. In this case, the expert (DM) corrects results only in the final stages, when the response does not accurately describe the requirements for facility requirements. This approach allows us to obtain the most rational decision in the formation of multicriteria system.

The formation of a variety of pendulum routes in the mathematical model represents a system of constrains allowing to provide all passenger flows $Q_{i}$ between points $i$-j, bandwidth limitations of SP and available number of passenger vehicles. The objective function should provide the greatest social and economic efficiency, which are reduced, usually to minimum turnaround time for traffic and to the shortest path between points of departure and destination. Next we make calculations and obtained data, in case of positive results, are represented to the decision maker. If you are not satisfied for any parameters, then DM is given the opportunity to impose restrictions on an unsatisfactory option in the general system of equation, and the procedure starts again. If the decision maker makes a decision about the appropriateness of the inclusion of the route in the network $G$, then it excludes the volume of passengers, which was adopted by the serving route. The calculations are repeated until all passenger traffics $Q_{i j}$ will be satisfied.

\section{Results and Discussion}

In the process of forming route network of passenger vehicles in cities and at its final adjustments for each route, the issues of increasing socio-economic efficiency together with the issues of choosing the number and type of vehicles on the route of CRN should be solved.

The number and types of passenger vehicles required for a specific route can be defined on the simplex method of solving linear programming. For the objective function in this case, it is advisable to use the function of minimizing the route cost, i.e.

$$
F(n) \sum C_{m} n_{m},
$$

where, $c_{1} \ldots c_{n}$ - minimal financial costs for the appropriate type of passenger vehicles for the maximum hour of traffic flow on this route during the reporting period of the day; $n$ $\ldots n_{m}$ - variables characterizing the number of rolling stock in the line of a certain kind and type. 
The task of formation of circular and pendulum routes is aimed for minimizing the download of $\mathrm{CRN}$, providing transportation of passengers and reducing pollution of environment from the vehicles of road passenger transport.

The number of passenger vehicles of each type is determined based on the maximum passenger flow. Limitation in this case is the minimum possible number of passenger vehicles in line with the maximum traffic interval, that is,

$$
[N]=\frac{60 l_{m}}{V_{s}[J]},
$$

Where, $l_{m}$ - route length, $\mathrm{km} ; V_{s}$ - average speed of passenger vehicles of the selected type, $\mathrm{km} / \mathrm{h} ;[]-$ maximum traffic interval during the period under review, min.

In this case the following inequality is used

$$
n_{1}+n_{2}+\ldots+n_{m} \geq[N]
$$

To provide the most complete transportation of passengers on the route it is necessary to consider the possibility of the carrier, i.e. the number and type of passenger vehicles which are available on line.

$$
\left\{\begin{array}{l}
n_{1} \leq N_{1} \\
n_{2} \leq N_{2} \\
n_{m} \ldots N_{m}
\end{array}\right.
$$

Selection of the type and number of passenger vehicles should be limited by maximum and minimum traffic flow, which must be served on specific routes of CRN, that is

$$
\begin{aligned}
& \left\{\begin{array}{l}
q_{1 n_{1}}+q_{2 n_{2}}+\ldots+q_{m} n_{m} \leq\left[q_{1}\right] \\
q_{1 n_{1}}+q_{2 n_{2}}+\ldots+q_{m} n_{m} \geq\left[q_{1}\right]
\end{array}\right. \\
& n_{1} \leq N_{1} \\
& n_{2} \leq N_{2}
\end{aligned}
$$

where $q_{1} \ldots q_{m}$ - capacity of passenger vehicles, pass; $\left[q_{1}\right]$ and $\left[q_{2}\right]-$ maximum and minimum possible passenger traffic on routes of CRN according to the studied daily time interval.

Value $\left[q_{1}\right]$ can be get, taken into intra-hour coefficient of irregularity of passenger flow, that is $\left[q_{1}\right]=\left[q_{2}\right] k$.

In the absence of data on the coefficient of intra-hour irregularity of passenger flow, it can be assumed to be equal to $K=1.1$.

Environmental requirements for passenger transport are determined by given values of maximum permissible concentration of toxic substances taking into account the exhaust gas of engines. The results of environmental measurements for each of the passenger transport are then compared with the maximum permissible concentration of toxic substances and adjustment to the vehicles to reduce emissions of harmful substances are made $B_{m}$ if they do not meet the normative requirements, that is,

$$
B_{1 n_{1}}+B_{2 n_{2}}+\ldots+B_{m} n_{m} \leq[B]
$$

Thus, the system of linear equations, describing the basic conditions and requirements for the formation of route transport network can be represented in the following form: 


$$
\begin{aligned}
& n_{1} \leq N_{1} \\
& n_{2} \leq N_{2} \\
& \ldots \ldots \ldots \\
& n_{m} \leq N_{m} \\
& B_{1 n_{1}}+B_{2 n_{2}}+\ldots+B_{m} n_{m} \leq[B] \\
& q_{1 n_{1}}+q_{2 n_{2}}+\ldots+q_{m} n_{m} \leq\left[q_{1}\right] \\
& q_{1 n_{1}}+q_{2 n_{2}}+\ldots+q_{m} n_{m} \geq\left[q_{1}\right] \\
& n_{1}+n_{2}+\ldots+n_{m} \geq[N] \\
& n_{1} \geq 0 \\
& n_{2} \geq 0 \\
& \ldots \ldots \ldots \\
& n_{m} \geq 0
\end{aligned}
$$

In this case, the objective function will be the minimum cost on routes of CRT that can be represented in the form

$$
F(n)=C_{1} n_{1}+C_{2} n_{2}+\ldots+C_{m} n_{m} \rightarrow \min ,
$$

Task solution of forming a route of transport network of a city comes before obtaining the optimal values of objective function (15). If the solution is not optimal one, then, for example, by changing basic variables $n_{1} \ldots n_{m}$ or making a transition to a new inequality, the following solution with a smaller value of the objective function is defined.

\section{Conclusion}

As a result of calculations after a finite number of steps, an optimal plan for the formation of route transport network of a city is formed with the use of circular and pendulum routes.

\section{References}

1. S. V. Belokurov, S. V. Velichko, D. E. Solovei, Synthesis of choice functions on the integration of search in numerical models of multicriteria optimization. 2nd edition. Voronezh: publishing house of Voronezh State University, P.125 (2004)

2. S. V. Belokurov, V. P. Belokurov, Models of multicriteria phased array in the information systems of management of transport processes. Transport, Science, Technology, Management. Scientific. Inform. B. of the Russian Academy of Sciences. Moscow: VINITI, 8, pp. 11-14 (2009)

3. S. V. Belokurov, S. V. Skryl, V. P. Belokurov, Peculiarities of model of optimum control of the process of elimination decisions based on the synthesis of theory choice in transport systems. Transport, Science, Technology, Management. Scientific. Inform. B. of the Russian Academy of Sciences. Moscow: VINITI, 1, pp. 5-9 (2010)

4. S. V. Belokurov, S. V. Skryl, V. P. Belokurov, Decision-making for efficient management of transportation systems based on choices situations. Transport, Science, 
Technology, Management. Scientific. Inform. B. of the Russian Academy of Sciences. Moscow: VINITI, 2, pp. 6-12 (2010)

5. V. V. Belyakov, M. E. Bushueva, V. I. Sagunov, Multicriteria optimization in the evaluation of mobility, competitiveness of automotive engineering and diagnostics of complex technical systems. N. Novgorod : NNSTU, P. 271 (2001)

6. N. I. Glebov, Yu. A. Kochetov, A.V. Plyasunov, Methods of optimization. Novosibirsk: NSU, P. 105 (2000)

7. A.V. Gnedin, An Efficient stop on the Pareto-optimal variant . Automation and Telemechanics, 3, pp.87-95 (1983)

8. N. Christofides, Graph theory. An algorithmic approach, M.: Mir, P. 432 (1978)

9. O. I. Larichev, Theory and methods of decision making, M.: Logos, 296 pp. (2000)

10. V. V. Podinovski, V. D. Nogin, Pareto-optimal solutions of multicriteria problems, M.: Science, 250 pp. (1982)

11. S.V. Belokurov, V.P. Belokurov, V.K. Zolnikov, O.N. Cerkasov, Methods of MultiCriteria Optimization in Problems of Simulation of Trucking Industry, Transformation Research Procedia, 20, pp. 47-52 (2017)

12. P. Kall, S. W. Wallance, Stochastic Programming . John Wiley and Sons, Chichester, 317 p. (1994)

13. X. Shen, X.Yao, Mathematical Modeling and Multiobjective Evolutionary Algorithms Applied to Dynamic Flexible Job Shop Scheduling Problems, Information Scienees, 298, pp. 198-224 (2015) 